\title{
Biomimetic Total Synthesis of (+)-Chloropupukeananin, (-)-Chloropupukeanolide D, and Chloropestolides
}

Takahiro Suzuki ${ }^{1, *}$, Soichiro Watanabe ${ }^{1}$, Wataru Ikeda $^{1}$, Susumu Kobayashi ${ }^{2}$ and Keiji Tanino ${ }^{1}$ ${ }^{1}$ Department of Chemistry, Hokkaido University, Sapporo, Hokkaido 060-0810, Japan

${ }^{2}$ Faculty of Pharmaceutical Sciences, Tokyo University of Science, Noda, Chiba 278-8510, Japan *Email: takahiro-suzuki@sci.hokudai.ac.jp

Abstract

Diels-Alderases have attracted the interest of the scientific community because of their potential as a universal molecular tool for the production of bioactive compounds. Although several Diels-Alderases are known to catalyse the intramolecular Diels-Alder reactions, it is urgent to discover Diels-Alderases that can catalyse intermolecular heterodimeric Diels-Alder reactions. The biosynthesis of chloropupukeananin, chloropupukeanolides, and chloropestolides is triggered by the intermolecular Diels-Alder reaction between (+)-iso-A82775C and (-)-maldoxin; however, the involvement of enzymes in this Diels-Alder reaction is not yet identified. We herein report the biomimetic total synthesis of $(+)$-chloropupukeananin, which proceeds via the Diels-Alder/carbonylene cascade reaction and the migration of the $p$-orsellinate group. Moreover, the effect of solvent on the intermolecular Diels-Alder reaction of siccayne and (-)-maldoxin to give chloropestolides $\mathrm{H}-\mathrm{K}$ suggested that the biosynthesis of the chloropupukeananin family involves a Diels-Alderase that catalyses the heterodimeric Diels-Alder reaction.

Natural products owe their structural diversity to enzymes. Enzymes, such as prenyl transferases ${ }^{1,2}$ and terpene cyclases, ${ }^{3}$ which catalyse the carbon-carbon bond formation and cyclisation reactions, are responsible for the diversity in the carbon skeleton of natural products. The Diels-Alder reaction, in which a cyclohexene ring can be constructed from diene and dienophile units via a concerted transition state by forming two $\mathrm{C}-\mathrm{C}$ bonds, is one of the most powerful tools for introducing molecular complexity in terms of biosynthesis, ${ }^{4,5,6,7}$ as well as chemical synthesis. ${ }^{8}$ There are many natural products that can be synthesised using the Diels-Alder reaction. However, most of them were proven to be just artefacts or products of spontaneous cycloaddition. ${ }^{9,10,11}$ Since the discovery of a Diels-Alderase in the biosynthesis of solanapyrone, ${ }^{12}$ only a few classes of this enzyme catalysing intramolecular reactions in polyketide synthesis ${ }^{13,14,15,16}$ have been identified. 
Very recently, enzymes promoting the intermolecular Diels-Alder reactions to produce pseudodimeric resveratrols have been identified. ${ }^{17}$ Meanwhile, artificial Diels-Alderases, such as antibody catalysts, ${ }^{18}$ artificial enzymes, ${ }^{19}$ and super molecules,${ }^{20,21,22}$ have been developed for realising intermolecular Diels-Alder reactions because of the high synthetic convergence and broad diversity of products. If an intermolecular heterodimeric Diels-Alderase is identified, its genetic modifications can be expected to pave the way for the versatile production of bioactive natural product-like compounds. $^{23}$

Chloropupukeananin (1), an inhibitor of HIV-1 replication, was originally isolated from the plant endophytic fungus Pestalotiopsis fici by Che et al. as the first chlorinated pupukeanane derivative (Figure 1a). ${ }^{24}$ Around that time, iso-A82775C (2) and pestheic acid (3) ${ }^{25,26,27}$ were also isolated and were proposed as the biosynthetic precursors of $\mathbf{1}$. One of the prominent structural features of 1 is the highly functionalised tricyclo[4.3.1. $0^{3,7}$ decane moiety that bore the vinyl allene moiety of $\mathbf{2}$ and the methyl benzoate moiety of $\mathbf{3}$. Thus, we speculated that $(R)$-maldoxin (4), which was generated by the asymmetric oxidative dearomatisation of $\mathbf{3}$, was the actual biosynthetic precursor instead of $3 .^{28}$ Following this, an intermolecular reverse-electron-demand Diels-Alder reaction between $\mathbf{2}$ and $\mathbf{4}$ and an intramolecular carbonyl-ene reaction led to the construction of the tricyclo[4.3.1. $\left.0^{3,7}\right]$ decane skeleton. Finally, the migration of the $p$-orsellinate moiety gave $\mathbf{1}$. Continuous efforts by Che et al. to unveil the biosynthetic pathway of 1 led to the identification of many natural heterodimeric congeners. These included (i) chloropestolides A-F (5-10), ${ }^{29,30}$ the isomers of the reverse- and normal-electron-demand Diels-Alder reactions, (ii) chloropupukeanolide $\mathrm{D}$ and $\mathrm{C}(\mathbf{1 1}, \mathbf{1 2}),{ }^{31}$ a carbonyl-ene product of $\mathbf{7}$ and $\mathbf{8}$, and (iii) other degradation products (Supplementary Fig. 1). Finally, optically active $(R)-4,{ }^{32}$ which was the missing link in the biosynthesis of $\mathbf{1}$, was isolated from the related fungus, $P$. theae. These results support our hypothesis on the biosynthesis of $\mathbf{1}$. Chloropestolides H-K (13-16) ${ }^{33}$ were also isolated during the identification of the biosynthetic gene cluster of 2 using a prenyl transferase gene-disrupted strain of a chloropupukeananin-producing bacterium. By inhibiting the biosynthesis of $\mathbf{2}$, intermediate siccayne (17) was found to undergo an intermolecular Diels-Alder reaction with 4 to afford four cycloadducts, 13-16. 


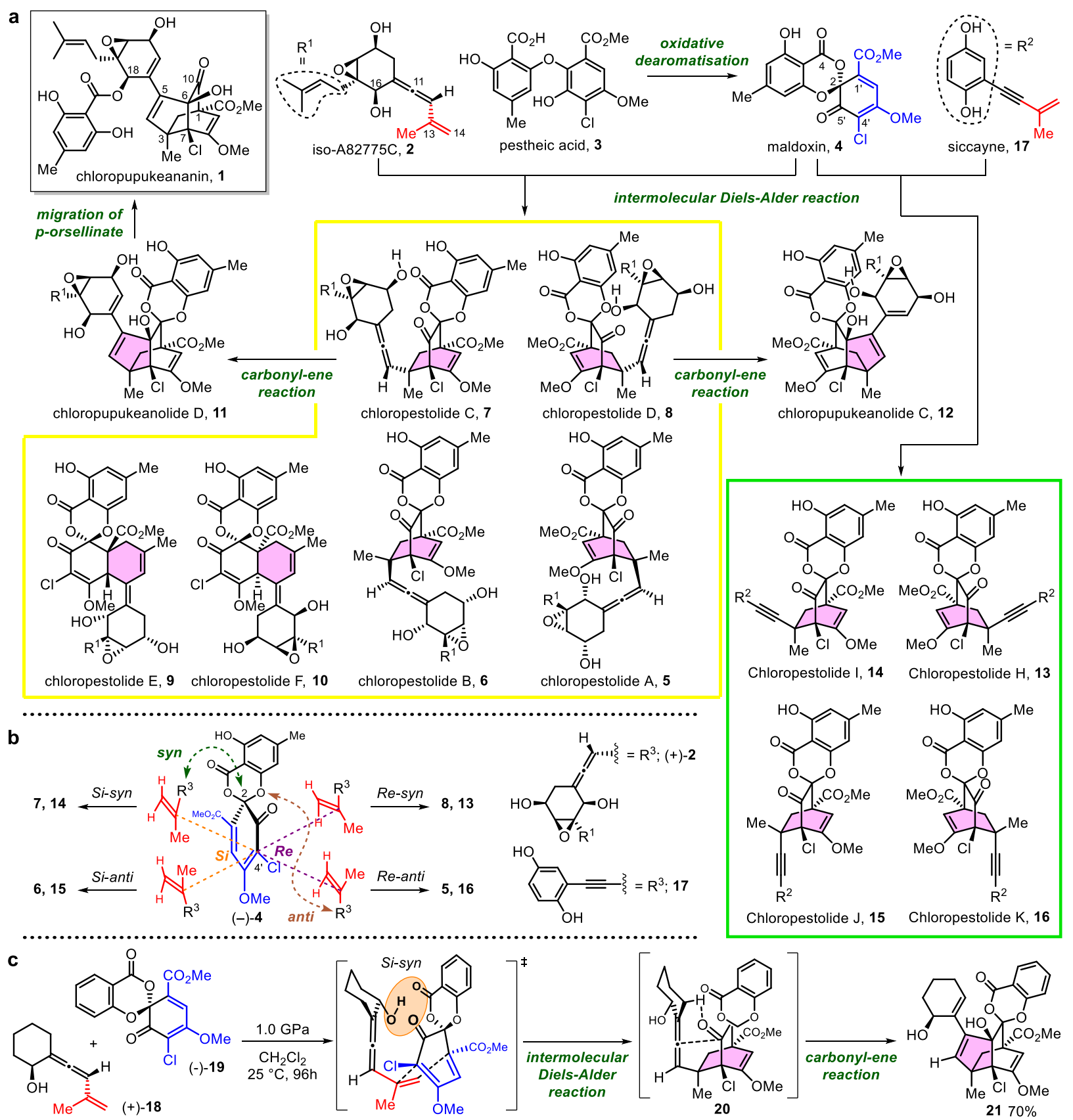

Fig. 1 | Chemical structure of the chloropupukeananin family and the facial selectivity of Diels-Alder reactions. a.

Proposed biosynthetic pathway of the chloropupukeananin family (Diels-Alder adducts from $\mathbf{2}$ and $\mathbf{4}$ are framed in yellow box and those from $\mathbf{4}$ and $\mathbf{1 7}$ are framed in green box). b. Four orientations of the diene and dienophile moieties in the reverse-electron-demand Diels-Alder reaction. c. Si-syn selectivity probably directed by the hydrogen bonding networks (highlighted in orange; our previous study). Diene and dienophile moieties are coloured in blue and red, respectively. The carbocycles formed by the Diels-Alder reactions or carbonyl-ene reactions are highlighted in pink. 
Isolation of a family of chloropestolides (5-8, 13-16) indicated poor facial selectivity of the intermolecular Diels-Alder reaction in the biosynthesis (Fig. 1b). Briefly, during the formation of these bicyclo[2.2.2] octane-containing cycloadducts, the dienophiles approached the Re- or $S i$-face of $\mathrm{C} 4$ ' of diene 4, with syn- or anti-orientation between the $\mathrm{R}^{3}$ group of the dienophiles and the $\mathrm{C} 2$ acetal moiety of 4 . The isolated amounts of 13-16 were 2.1, 6.4, 2.2, and $1.6 \mathrm{mg}$, respectively (13:14:15:16 ratio was 17:52:18:13). ${ }^{33}$ The intermolecular Diels-Alder reaction gave all possible cycloadducts, although with some Si-syn selectivity. The diversity of $\mathbf{1}$ and its related compounds arises from the poor selectivity in Diels-Alder reaction, in addition to the highly functionalised and complex structure of the resulting bicyclo[2.2.2] octanes that undergo subsequent reactions, including carbonyl-ene reactions and/or other degradation reactions. In contrast, our previous synthetic study on chloropupukeananin ${ }^{34}$ demonstrated the selective construction of a tricyclo[4.3.1.0 $\left.0^{3,7}\right]$ decane skeleton under high pressure conditions (Fig. 1c). Intermolecular Diels-Alder/carbonyl-ene cascade reaction of model compounds (+)-18 and (-)-19 gave tricyclic compound $\mathbf{2 1}$ in 70\% yield via DielsAlder adduct 20. The Si-syn transition state was probably stabilised by hydrogen bonding networks. These indicated that the intermolecular Diels-Alder reactions of $\mathbf{4}$ in nature proceeded with poor selectivity, whereas those in chemical synthesis proceeded selectively. This was contradictory to the usual enzyme reactions in biosynthesis. Moreover, if these were enzyme reactions, the enzyme might be the first example of an intermolecular heterodimeric Diels-Alderase that could construct a highly functionalised bicyclo[2.2.2] octane skeleton. Inspired by the biosynthetic origin of this class of compounds, in addition to their structural complexity, we investigated the total synthesis of $\mathbf{1}$ and its related natural products. We herein report the biomimetic total synthesis of $(+)-\mathbf{1}$ using $(+)$-isoA82775C and (-)-maldoxin. The synthesis involved a Diels-Alder/carbonyl-ene cascade reaction and the migration of the $p$-orsellinate group. The effect of solvent on the Diels-Alder reaction of $(-)$ maldoxin suggested that the biosynthesis of the chloropupukeananin family involved a DielsAlderase that catalysed an intermolecular heterodimeric Diels-Alder reaction.

\section{Results and discussion}

Compounds (+)-2 and (-)-4 were already synthesised by us previously (summary in Supplementary Fig. 2 and 3). ${ }^{35,36}$ However, there are some limitations with regard to the scale-up synthesis for conducting further studies (Fig. $2 \mathrm{a}$ and $2 \mathrm{~b}$ ). The point of concern in the total synthesis of $(+)$-2 from 4-bromo-3-hydroxypyrone 22 is the low conversion $(\sim 30 \%)$ in the anti-S $\mathrm{S}_{\mathrm{N}} 2$ ' reaction of propargyl chloride $\mathbf{2 3}$ using a vinyl copper reagent. This problem was addressed by using an 
organoindium reagent in the presence of a Pd catalyst, ${ }^{37}$ and subsequently deprotecting the triethylsilyl (TES) groups of the resulting allene, which gave (+)-2 in 92\% yield at the sub-gram scale. In our previous study on the synthesis of (-)-4, phenol 27 was prepared from 5methoxysalicylic acid in five steps, one of which included non-selective chlorination. ${ }^{28}$ The alternative synthesis was initiated with 4-chloro-2,5-dimethoxybenzaldehyde $\mathbf{2 4}$, which was prepared by formylation of commercial 2-chloro-1,4-dimethoxybenzene. ${ }^{38}$ Pinnick oxidation and esterification of 24 gave salicylate $\mathbf{2 5}$ in $81 \%$ yield. Under Kita's conditions, ${ }^{39}$ the site-selective oxidation using (diacetoxyiodo)benzene (PIDA) in a trifluoroacetic acid (TFA)/acetic acid mixed solvent gave acetate 26, which underwent one-pot hydrolysis to give phenol 27 in 68\% yield. Phenol 27 was converted to (-)-4 according to our previously reported synthetic protocol. ${ }^{36}$

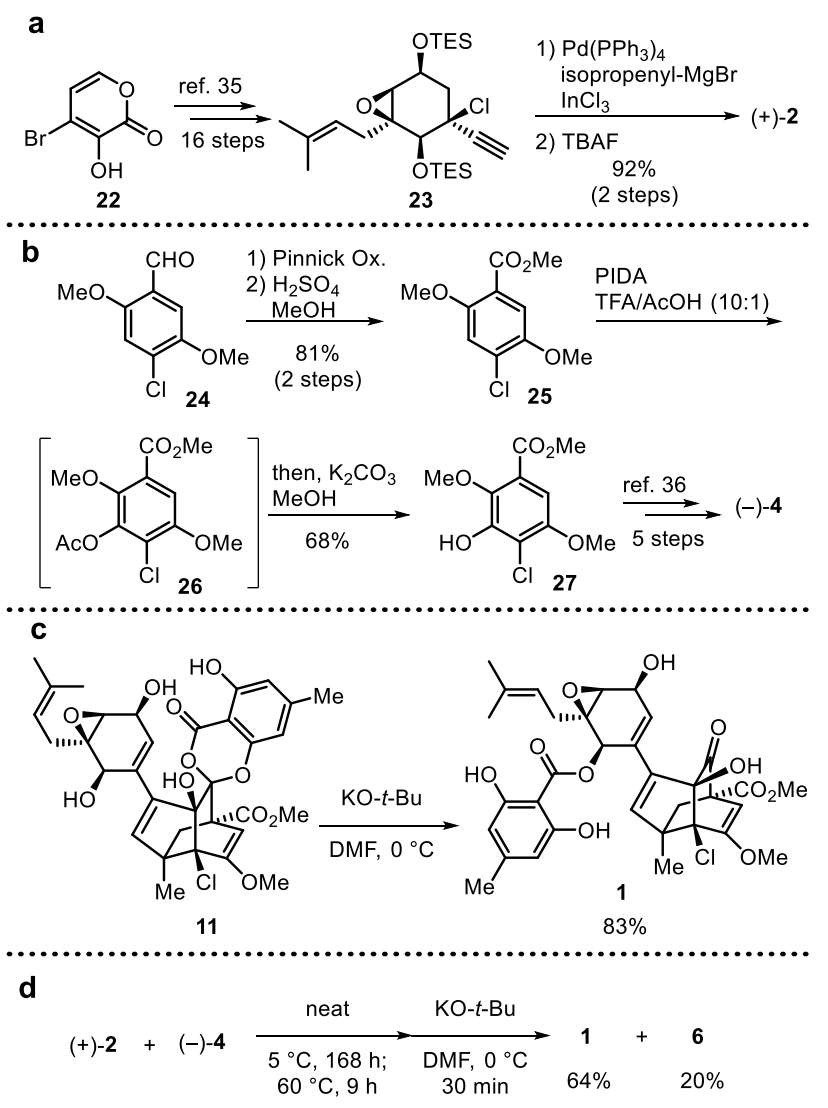

Fig. 2 Improved synthesis of (+)-iso-A82775C and (-)-maldoxin and total synthesis of chloropupukeananin. a.

Synthesis of 2 via the Pd-catalysed anti-S $\mathrm{S}_{\mathrm{N}} 2$ ' reaction using an organoindium reagent. $\mathbf{b}$. Alternate preparation of 27 via site-selective Kita oxidation. c. Migration of $p$-orsellinate group in the presence of potassium tert-butoxide. d. One-pot biomimetic total synthesis of chloropupukeananin. (TBAF: tetra- $n$-butylammonium fluoride; DMF: $N, N$ dimethylformamide). 
With the optically pure biosynthetic precursors (+)-2 and (-)-4 in hand, we investigated the intermolecular Diels-Alder/intramolecular carbonyl-ene cascade reaction, based on our proposed biosynthetic pathway. As in the model study, the mixture of (+)-2 and (-)-4 was subjected to a high pressure (Table 1). The intermolecular Diels-Alder reaction was almost complete in $1 \mathrm{~h}$ (entry 1 ), and the desired Si-syn cycloadduct 7 and one diastereomer, Si-anti cycloadduct 6, were obtained. Notably, no other cycloadduct was detected, as in our previous study, except for a small amount of carbonyl-ene product 11. Prolonging the reaction time facilitated the intramolecular carbonyl-ene reaction; when the reaction was conducted for $64 \mathrm{~h}$ (entry 4), 11 was obtained in $71 \%$ yield. Since the mixture of $\mathbf{7}$ and $\mathbf{1 1}$ were inseparable, the mixture was heated under atmospheric pressure (AP), so that the intramolecular carbonyl-ene reaction reaches completion. After the reaction under highpressure conditions (entry 5; $1.0 \mathrm{GPa}, 64 \mathrm{~h}$ ), the reaction mixture was concentrated and heated to $60{ }^{\circ} \mathrm{C}$ to afford 11 and 6 in $69 \%$ and $21 \%$ isolated yields, respectively. We investigated the conditions of the Diels-Alder reaction under atmospheric pressure. Heating the mixture of (+)-2 and (-)-4 at $70{ }^{\circ} \mathrm{C}$ in 1,2-dichloroethane gave a mixture of cycloadducts $\mathbf{6 , 7}$, and $\mathbf{1 1}$ in low yield due to the decomposition of the starting materials (entry 6). Next, the mixture of (+)-2 and (-)-4 was allowed to react under neat conditions at $25^{\circ} \mathrm{C}$ for $120 \mathrm{~h}$ (entry 7). After the completion of the intermolecular Diels-Alder reaction, the mixture was heated to $60{ }^{\circ} \mathrm{C}$ to give the desired compound $\mathbf{1 1}$ in $57 \%$ yield, along with 6 in $25 \%$ yield. These results indicated that a high pressure significantly accelerated the intermolecular Diels-Alder reactions but did not affect the facial selectivity. For the selectivity, we postulated that the hydrogen bonding networks between the C16-alcohol group of (+)-2 and the two carbonyl oxygen atoms (at C4 and C5') of (-)-4 stabilised the Si-syn transition state giving the desired cycloadduct 7 (Fig. 3). On the other hand, the C16-alcohol group of (+)-2 could only form a single hydrogen bond with an oxygen atom of the C3'-methoxy group (Si-anti and Re-anti) or C5'ketone group (Re-syn) of (-)-4 in the other transition states. 

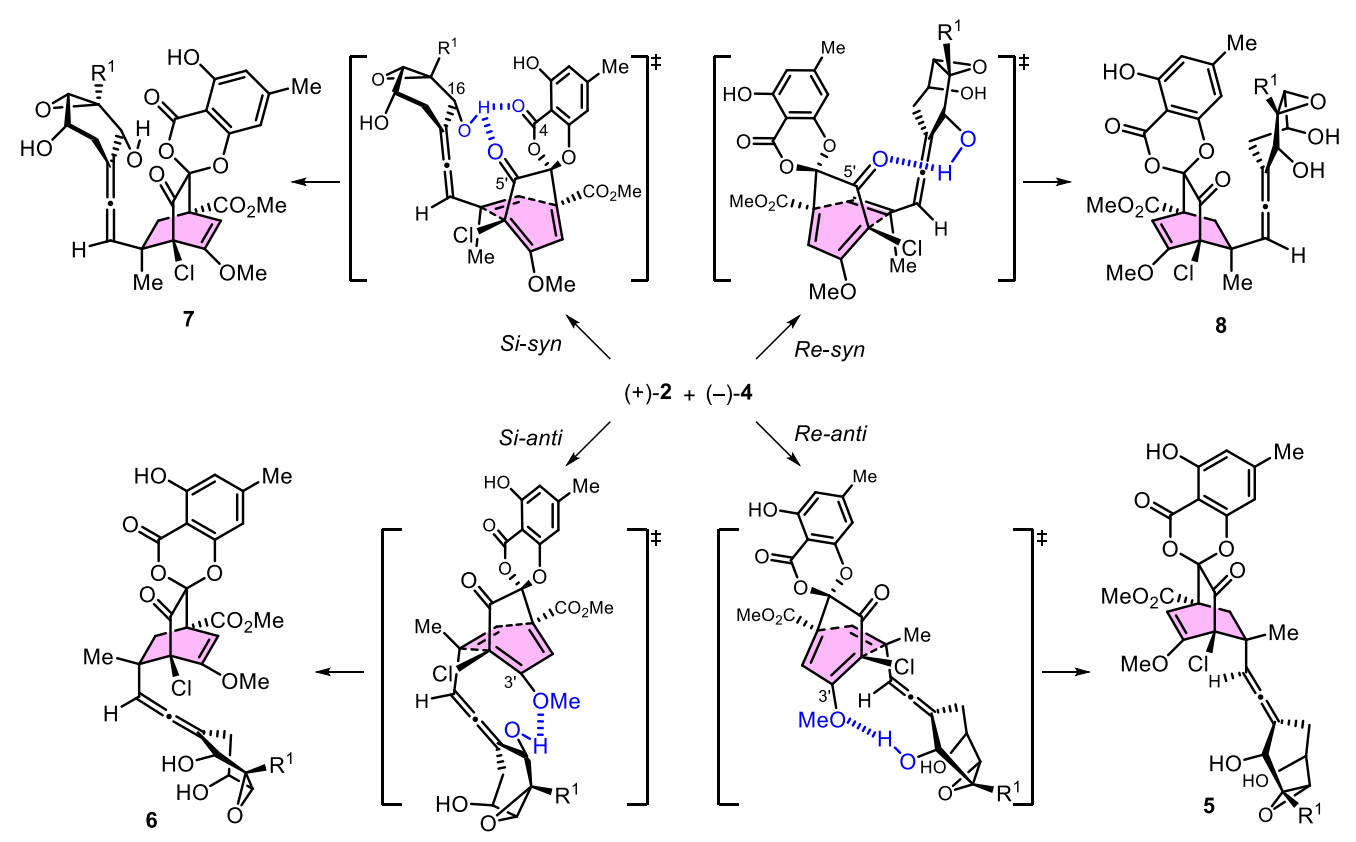

Fig. 3 | Probable transition state of the intermolecular Diels-Alder reaction between (+)-2 and (-)-4. The formed cyclohexene rings are highlighted in red and intermolecular hydrogen bonds are highlighted in blue.

Finally, treatment with $\mathrm{KO} t$ - $\mathrm{Bu}$ in $\mathrm{DMF}$ resulted in the migration of the $p$-orsellinate group of 11, affording 1 in 83\% yield (Fig. 2c). Because the Diels-Alder/carbonyl-ene cascade reaction did not require any reagents, we attempted the one-pot biomimetic transformation from (+)-2 and (-)-4. The Diels-Alder/carbonyl-ene cascade reaction under AP ((i)neat, $5{ }^{\circ} \mathrm{C}, 168 \mathrm{~h}$; (ii) $60{ }^{\circ} \mathrm{C}, 9 \mathrm{~h}$ ) and the following migration reaction using $\mathrm{KO} t$-Bu in DMF provided 1 in $64 \%$ yield, along with 6 in $20 \%$ yield (Fig. 2d). Thus, the first asymmetric total synthesis of chloropupukeananin was accomplished. The spectral data of $\mathbf{1 , 6}$, and $\mathbf{1 1}$ were identical to those of the corresponding natural compounds. ${ }^{24,30,31}$

The current synthesis demonstrates the intriguing face selectivity of the intermolecular Diels-Alder reaction between (+)-2 and (-)-4; i.e., only two cycloadducts, 6 and 7, were obtained in the chemical synthesis, while four types of reverse-electron-demand cycloadducts 5-8 and two normal-electron-demand cycloadducts $\mathbf{9}$ and $\mathbf{1 0}$ were isolated as natural products. ${ }^{29,30}$ This suggests the involvement of enzymes in the intermolecular Diels-Alder reaction in biosynthesis; the enzymes activate the substrates to give diverse Diels-Alder products. However, it is also possible that cycloadducts 6 and 7 were obtained as major products, without the involvement of enzymes in the biosynthesis. The subsequent reactions, such as the carbonyl-ene reaction or other degradation 
reactions, consumed most of $\mathbf{6}$ and 7 with or without involving enzymes. Consequently, all the cycloaddition products, 5-10, were isolated apparently in a non-selective manner. Therefore, a simple system to directly compare the cycloadducts of the chemical synthesis with the natural cycloadducts is required.

To this end, we investigated the intermolecular Diels-Alder reaction of siccayne $(\mathbf{1 7})^{40}$ and (-)-maldoxin 4 in the biosynthesis of chloropestolides H-K (Table 2). First, the mixture of 17 and $(-)-4$, without a solvent, was allowed to stand for $24 \mathrm{~h}$ at room temperature (entry 1 ) to allow the Diels-Alder reaction to reach completion; this gave a mixture of 13-16 (13:14:15:16 yield ratio $=$ 9:32:51:8) in quantitative yield. Owing to the nature of $(-)-4,{ }^{36}$ a preference for the $\mathrm{Si}$-face was observed, as expected. Next, the Diels-Alder reaction of $\mathbf{1 7}$ and (-)-4 in $\mathrm{MeOH} / \mathrm{H}_{2} \mathrm{O}$ (entry 2) gave a mixture containing predominantly Si-anti $15(13: 14: 15: 16=4: 11: 74: 11)$, which was incompatible with the Si-syn selectivity of isolation. It is surprising that the product ratio in the aqueous solvent differed from that of the isolated sample; generally, hydrophilic solvents can simulate the reaction conditions for enzymatic reactions. Next, we focused on the relationship between the product ratio and solvents to reproduce the isolated ratio of 13-16. After investigating the Diels-Alder reaction using common organic solvents (entries 3-10; the entire solvent screening data are given in Supplementary Table 1), we found that the ratio, especially 14:15, depended on the solvent basicity (SB) ${ }^{41,42}$ and not on the dielectric constant $(\varepsilon)$, dipole moment $(\mu)$, or solvent polarity parameter $\left(\mathrm{E}_{\mathrm{T}}{ }^{\mathrm{N}}\right) .{ }^{43}$ In solvents with low basicity $(\mathrm{SB}<0.2), \mathbf{1 4}$ and 15 were obtained in nearly 1:1 ratio. The best result for the $\mathrm{Si}$-syn selectivity was obtained in $\mathrm{CH}_{2} \mathrm{Cl}_{2}$ (entry 4), giving $\mathbf{1 4}$ and $\mathbf{1 5}$ in $39 \%$ and $34 \%$ isolated yields, respectively. On the other hand, in solvents with high basicity ( $\mathrm{SB}>0.2)$, the Diels-Alder reaction gave predominantly 15. Moreover, the same trends were observed under highpressure conditions (entries 11 and 12). Comparing the ratio of 13-16 isolated from nature ${ }^{33}$ revealed that the preference for $\mathbf{1 4}$ was not reproduced in any organic solvents. Moreover, comparatively similar results were obtained in lipophilic solvents (entries 3 and 4) rather than in hydrophilic solvents (entries 2 and 9). Thus, there was no possibility that cycloadducts 13-16 were artefacts during cell cultivation and isolation. These facts suggest that the biosynthesis of 13-16 involves a heterodimeric Diels-Alderase that catalyses the intermolecular Diels-Alder reaction of $(+)-2$ and $(-)$ 4. 
In conclusion, the biomimetic total synthesis of $(+)$-chloropupukeananin (overall $4.5 \%$ yield, 19 steps from pyrone 22) and (-)-chloropupukeanolide D via an intermolecular Diels-Alder reaction using (-)-maldoxin and (+)-iso-A82775C and an intramolecular carbonyl-ene reaction has been achieved. Moreover, the biomimetic synthesis of chloropestolides H-K using (-)-maldoxin and siccayne unveiled the solvent effect of the intermolecular Diels-Alder reaction. The origin of the stereoselectivity of the intermolecular Diels-Alder reaction is still elusive; however, the biosynthesis of the chloropupukeananin family should involve enzymes that catalyse the intermolecular DielsAlder reaction. Results of the computational analysis of the facial selectivity of the intermolecular Diels-Alder reaction will be reported in due course.

\section{Methods}

Materials, methods, and characterisation data for all experiments are described in detail in the Supplementary Information.

\section{Acknowledgements}

This research was supported in part by JSPS KAKENHI grant number JP15H05842 in the Middle Molecular Strategy, JP18H01970 in Grant-in-Aid for Scientific Research(B), JP20K05485 in Grantin-Aid for Scientific Research(C), and the Photo-excitonix Project in Hokkaido University. We thank Dr. M. Sodeoka and Dr. S. Kawamura (RIKEN) for assistance with the operation of the high-pressure apparatus. We also thank the Naito Foundation, the Uehara Memorial Foundation, and the Research Foundation for Pharmaceutical Sciences for their financial support.

\section{Author contributions}

T.S., S.K., and K.T. conceived and designed the project. T.S., S.W., and W.I. performed the synthesis and analysed the data. T.S. wrote the manuscript with input from all the authors.

\section{Competing interests}

The authors declare no competing interests. 


\section{References}

1 Liang, P. H., Ko, T. P. \& Wang, A. H. Structure, mechanism and function of prenyltransferases. Eur. J. Biochem. 269, 3339-3354, (2002).

2 Takahashi, S. \& Koyama, T. Structure and function of cis-prenyl chain elongating enzymes. Chem. Rec. 6, 194205, (2006).

3 Christianson, D. W. Structural and Chemical Biology of Terpenoid Cyclases. Chem. Rev. 117, 11570-11648, (2017).

Klas, K., Tsukamoto, S., Sherman, D. H. \& Williams, R. M. Natural Diels-Alderases: Elusive and Irresistable. J. Org. Chem. 80, 11672-11685, (2015).

Minami, A. \& Oikawa, H. Recent advances of Diels-Alderases involved in natural product biosynthesis. $J$. Antibiot. 69, 500-506, (2016).

Jeon, B. S., Wang, S. A., Ruszczycky, M. W. \& Liu, H. W. Natural [4 + 2]-Cyclases. Chem. Rev. 117, 5367-5388, (2017).

Jamieson, C. S., Ohashi, M., Liu, F., Tang, Y. \& Houk, K. N. The expanding world of biosynthetic pericyclases: cooperation of experiment and theory for discovery. Nat. Prod. Rep. 36, 698-713, (2019).

Nicolaou, K. C., Snyder, S. A., Montagnon, T. \& Vassilikogiannakis, G. The Diels-Alder Reaction in Total Synthesis. Angew. Chem. Int. Ed. 41, 1668-1698, (2002).

Gravel, E. \& Poupon, E. Biogenesis and Biomimetic Chemistry: Can Complex Natural Products Be Assembled Spontaneously? Eur. J. Org. Chem. 2008, 27-42, (2007).

Wang, T. \& Hoye, T. R. Diels-Alderase-free, bis-pericyclic, [4+2] dimerization in the biosynthesis of (+/-)paracaseolide A. Nat Chem 7, (2015).

Godfrey, R. C., Green, N. J., Nichol, G. S. \& Lawrence, A. L. Total synthesis of brevianamide A. Nat Chem 12, 615-619, (2020).

Oikawa, H., Suzuki, Y., Naya, A., Katayama, K. \& Ichihara, A. First Direct Evidence in Biological Diels-Alder Reaction of Incorporation of Diene-Dienophile Precursors in the Biosynthesis of Solanapyrones. J. Am. Chem. Soc. 116, 3605-3606, (1994).

Auclair, K. et al. Lovastatin Nonaketide Synthase Catalyzes an Intramolecular Diels-Alder Reaction of a Substrate Analogue. J. Am. Chem. Soc. 122, 11519-11520, (2000).

Hashimoto, T. et al. Biosynthesis of versipelostatin: identification of an enzyme-catalyzed [4+2]-cycloaddition required for macrocyclization of spirotetronate-containing polyketides. J. Am. Chem. Soc. 137, 572-575, (2015). Byrne, M. J. et al. The Catalytic Mechanism of a Natural Diels-Alderase Revealed in Molecular Detail. J. Am. Chem. Soc. 138, 6095-6098, (2016).

Kim, H. J., Ruszczycky, M. W., Choi, S. H., Liu, Y. N. \& Liu, H. W. Enzyme-catalysed [4+2] cycloaddition is a key step in the biosynthesis of spinosyn A. Nature 473, 109-112, (2011).

Gao, L. et al. FAD-dependent enzyme-catalysed intermolecular [4+2] cycloaddition in natural product biosynthesis. Nat. Chem. 12, 620-628, (2020). 
Science 262, 204-208, (1993).

19 Siegel, J. B. et al. Computational design of an enzyme catalyst for a stereoselective bimolecular Diels-Alder reaction. Science 329, 309-313, (2010).

Kang, J. \& Rebek, J., Jr. Acceleration of a Diels-Alder reaction by a self-assembled molecular capsule. Nature 385, 50-52, (1997).

21 Yoshizawa, M., Tamura, M. \& Fujita, M. Diels-Alder in aqueous molecular hosts: unusual regioselectivity and efficient catalysis. Science 312, 251-254, (2006).

Palma, A. et al. Cucurbit[7]uril as a Supramolecular Artificial Enzyme for Diels-Alder Reactions. Angew. Chem. Int. Ed. 56, 15688-15692, (2017).

Basler, S. et al. Efficient Lewis acid catalysis of an abiological reaction in a de novo protein scaffold. Nat Chem 13, 231-235, (2021).

Liu, L. et al. Chloropupukeananin, the first chlorinated pupukeanane derivative, and its precursors from Pestalotiopsis fici. Org. Lett. 10, 1397-1400, (2008).

Shimada, A., Takahashi, I., Kawano, T. \& Kimura, Y. Chloroisosulochrin, Chloroisosulochrin Dehydrate, And Pestheic Acid, Plant Growth Regulators, Produced By Pestalotiopsis Theae. Z. Naturforsch. B 56, 797-803, (2001).

Ogawa, T. et al. RES-1214-1 and -2, novel non-peptidic endothelin type A receptor antagonists produced by Pestalotiopsis sp. J. Antibiot. 48, 1401-1406, (1995).

Adeboya, M. O. et al. Metabolites of the higher fungi. Part 29. Maldoxin, maldoxone, dihydromaldoxin, isodihydromaldoxin and dechlorodihydromaldoxin. A spirocyclohexadienone, a depsidone and three diphenyl ethers: keys in the depsidone biosynthetic pathway from a member of the fungus genus Xylaria. J. Chem. Soc., Perkin Trans. 1, 1419-1425, (1996).

28 Suzuki, T. \& Kobayashi, S. Concise approach to pupukeanane skeleton: synthetic study of chloropupukeananin. Org. Lett. 12, 2920-2923, (2010).

29 Liu, L. et al. Chloropestolide A, an antitumor metabolite with an unprecedented spiroketal skeleton from Pestalotiopsis fici. Org. Lett. 11, 2836-2839, (2009).

30 Liu, L. et al. Spiroketals of Pestalotiopsis fici provide evidence for a biosynthetic hypothesis involving diversified Diels-Alder reaction cascades. J. Org. Chem. 78, 2992-3000, (2013).

31 Liu, L. et al. Chloropupukeanolides C-E: cytotoxic pupukeanane chlorides with a spiroketal skeleton from Pestalotiopsis fici. Chem.-Eur. J. 17, 2604-2613, (2011).

32 Liu, L. et al. Chlorotheolides A and B, Spiroketals Generated via Diels-Alder Reactions in the Endophytic Fungus Pestalotiopsis theae. J. Nat. Prod. 79, 2616-2623, (2016).

33 Pan, Y. et al. Characterization of a Prenyltransferase for Iso-A82775C Biosynthesis and Generation of New Congeners of Chloropestolides. ACS Chem. Biol. 13, 703-711, (2018).

34 Suzuki, T. et al. Unexpected Diels-Alder/carbonyl-ene cascade toward the biomimetic synthesis of chloropupukeananin. Org. Lett. 15, 1748-1751, (2013).

35 Suzuki, T., Watanabe, S., Kobayashi, S. \& Tanino, K. Enantioselective Total Synthesis of (+)-Iso-A82775C, a Proposed Biosynthetic Precursor of Chloropupukeananin. Org. Lett. 19, 922-925, (2017). 
Chloropupukeananin Family. Org. Lett. 20, 3919-3922, (2018).

37 Riveiros, R., Rodriguez, D., Perez Sestelo, J. \& Sarandeses, L. A. Palladium-catalyzed cross-coupling reaction of triorganoindium reagents with propargylic esters. Org. Lett. 8, 1403-1406, (2006).

38 Bloomer, J. L., Stagliano, K. W., \& Gazzillo, J. A. Preparation of functionalized juglone acetates and juglones via 1,4-dimethoxynaphthalene derivatives: synthesis of anthraquinones related to rhein and aloe-emodin. J. Org. Chem., 58, 7906-7912, (1993).

39 Kita, Y. et al. Hypervalent Iodine-Induced Nucleophilic Substitution of para-Substituted Phenol Ethers. Generation of Cation Radicals as Reactive Intermediates. J. Am. Chem. Soc. 116, 3684-3691, (1994).

40 Pinault, M., Frangin, Y., Genet, J. P. \& Zamarlik, H. Total Synthesis of Siccayne. Synthesis 1990, 935-937, (1990).

41 Catalán, J., Palomar, J., Díaz, C. \& de Paz, J. L. G. On Solvent Basicity: Analysis of the SB Scale. J. Phys. Chem. A 101, 5183-5189, (1997).

42 Catalán, J. et al. A Generalized Solvent Basicity Scale: The Solvatochromism of 5-Nitroindoline and Its Homomorph 1-Methyl-5-nitroindoline. Liebigs Ann. Chem. 1996, 1785-1794, (1996).

43 Reichardt, C. \& Welton, T. Solvents and Solvent Effects in Organic Chemistry Fourth, Updated and Enlarged Edition (Wiley, 2011). 
Table 1 | Intermolecular Diels-Alder/carbonyl-ene cascade reactions under high pressure and atmospheric pressure
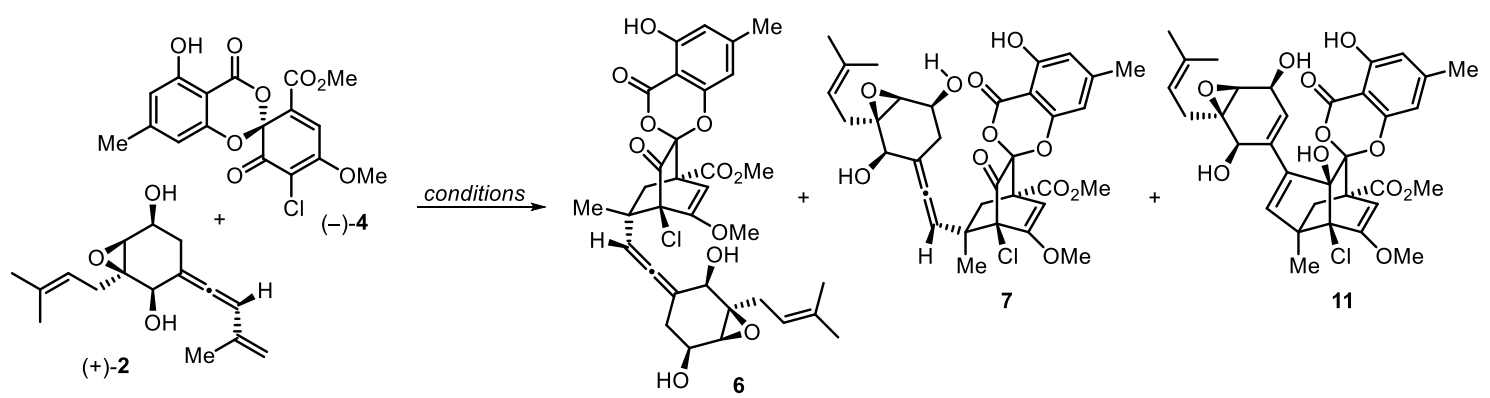

\begin{tabular}{|c|c|c|c|c|c|c|c|c|}
\hline \multirow[t]{2}{*}{ entry } & \multirow{2}{*}{$\begin{array}{c}(+)-2 \\
\text { (equiv.) }\end{array}$} & \multirow{2}{*}{$\begin{array}{l}(-)-4 \\
\text { (equiv.) }\end{array}$} & \multirow[t]{2}{*}{ reaction conditions } & \multirow{2}{*}{$\begin{array}{l}\text { temp. } \\
\left({ }^{\circ} \mathrm{C}\right)\end{array}$} & \multirow{2}{*}{$\begin{array}{l}\text { time } \\
\text { (h) }\end{array}$} & \multicolumn{3}{|c|}{ yield $(\%)$} \\
\hline & & & & & & 6 & 7 & 11 \\
\hline $1^{\mathrm{a}}$ & 1.3 & 1.0 & $1.0 \mathrm{GPa}, \mathrm{CH}_{2} \mathrm{Cl}_{2}$ & 25 & 1 & 17 & 67 & 3 \\
\hline $2^{\mathrm{a}}$ & 1.3 & 1.0 & 1.0 GPa, $\mathrm{CH}_{2} \mathrm{Cl}_{2}$ & 25 & 4 & 17 & 62 & 13 \\
\hline $3^{\mathrm{a}}$ & 1.3 & 1.0 & 1.0 GPa, $\mathrm{CH}_{2} \mathrm{Cl}_{2}$ & 25 & 16 & 17 & 42 & 40 \\
\hline $4^{\mathrm{a}}$ & 1.3 & 1.0 & 1.0 GPa, $\mathrm{CH}_{2} \mathrm{Cl}_{2}$ & 25 & 64 & 16 & 5 & 71 \\
\hline $5^{\mathrm{b}}$ & 1.3 & 1.0 & $\begin{array}{l}\text { (i) } 1.0 \mathrm{GPa}, \mathrm{CH}_{2} \mathrm{Cl}_{2} \\
\text { (ii) } \mathrm{AP} \text {, neat }\end{array}$ & $\begin{array}{l}25 \\
60\end{array}$ & $\begin{array}{l}64 \\
9\end{array}$ & 21 & - & 69 \\
\hline $6^{\mathrm{a}}$ & 1.0 & 1.5 & $\mathrm{AP},\left(\mathrm{CH}_{2} \mathrm{Cl}\right)_{2}$ & 70 & 30 & 21 & 13 & 19 \\
\hline $7^{\mathrm{b}}$ & 1.0 & 1.0 & $\begin{array}{l}\text { (i) AP, neat } \\
\text { (ii) AP, neat }\end{array}$ & $\begin{array}{l}25 \\
60\end{array}$ & $\begin{array}{l}120 \\
68\end{array}$ & 25 & - & 57 \\
\hline
\end{tabular}

${ }^{a}$ NMR yield. ${ }^{b}$ isolated yield. AP, atmospheric pressure. 
Table 2 | Facial selectivity of the intermolecular Diels-Alder reaction between maldoxin and siccayne.

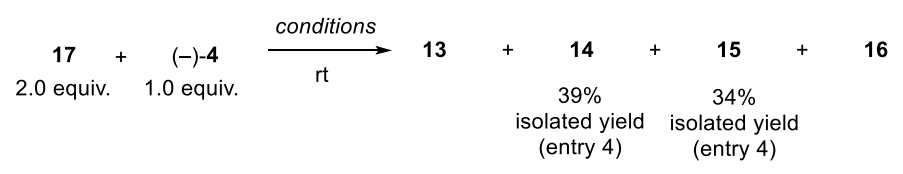

\begin{tabular}{|c|c|c|c|c|c|c|c|c|c|c|}
\hline \multirow[t]{2}{*}{ entry } & \multirow[t]{2}{*}{ reaction conditions } & \multirow[t]{2}{*}{ SB } & \multirow[t]{2}{*}{$\varepsilon_{\mathrm{r}}$} & \multirow{2}{*}{$\begin{array}{c}\mu \\
\left(10^{-30} \mathrm{Cm}\right)\end{array}$} & \multirow[t]{2}{*}{$\mathrm{E}_{\mathrm{T}}^{\mathrm{N}}$} & \multirow{2}{*}{$\begin{array}{l}\text { time } \\
\text { (h) }\end{array}$} & \multicolumn{4}{|c|}{ ratio $^{a}$} \\
\hline & & & & & & & 13 & 14 & 15 & 16 \\
\hline & isolation (ref. 33) & & & & & - & 17 & 52 & 18 & 13 \\
\hline $1^{\mathrm{b}}$ & neat & & & & & 24 & 9 & 32 & 51 & 8 \\
\hline 2 & $\mathrm{MeOH} / \mathrm{H}_{2} \mathrm{O}(4 / 1)$ & & & & & 48 & 4 & 11 & 74 & 11 \\
\hline 3 & benzene & 0.124 & 2.3 & 0 & 0.111 & 48 & 9 & 42 & 44 & 5 \\
\hline 4 & $\mathrm{CH}_{2} \mathrm{Cl}_{2}$ & 0.178 & 8.9 & 3.8 & 0.309 & 48 & 13 & 44 & 37 & 6 \\
\hline 5 & $\mathrm{CH}_{3} \mathrm{CN}$ & 0.286 & 37.5 & 13.0 & 0.460 & 48 & 10 & 28 & 53 & 9 \\
\hline 6 & acetone & 0.475 & 20.6 & 9.0 & 0.355 & 48 & 6 & 20 & 64 & 10 \\
\hline 7 & $\mathrm{MeOH}$ & 0.545 & 32.7 & 5.9 & 0.762 & 48 & 4 & 11 & 75 & 10 \\
\hline 8 & $\mathrm{Et}_{2} \mathrm{O}$ & 0.562 & 4.2 & 3.8 & 0.117 & 48 & 6 & 17 & 69 & 7 \\
\hline 9 & $\mathrm{EtOH}$ & 0.658 & 24.6 & 5.8 & 0.654 & 48 & 3 & 7 & 80 & 10 \\
\hline 10 & $i-\mathrm{PrOH}$ & 0.762 & 19.9 & 5.5 & 0.546 & 48 & 3 & 5 & 81 & 11 \\
\hline $11^{\mathrm{b}}$ & 1.0 GPa, MeOH/CH & $(10 / 1)$ & & & & 1 & 3 & 11 & 75 & 11 \\
\hline $12^{b}$ & 1.0 GPa, $\mathrm{CH}_{2} \mathrm{Cl}_{2}$ & & & & & 1 & 15 & 46 & 33 & 6 \\
\hline
\end{tabular}

${ }^{a}$ Ratio was determined by ${ }^{1} \mathrm{H}-\mathrm{NMR} .{ }^{\mathrm{b}} 1.1$ equiv. of $\mathbf{1 7}$ was used. 